\title{
UBV PHOTOMETRY OF V705 CAS (NOVA CAS 1993) DURING THE FIRST YEAR OF THE OUTBURST
}

\author{
K. PETRÍK ${ }^{1}$, L. HRIC ${ }^{2}$, Z. URBAN ${ }^{2}$, D. HANŽL $L^{3}$, \\ D. $\mathrm{CHOCHOL}^{2}$ \\ 1.Hlohovec Observatory, Sládkovičova 41, 92001 Hlohovec, \\ The Slovak Republic. e-mail: astropet@ta3.sk \\ 2. Astronomical Institute of the Slovak Academy of Sciences, \\ 05960 Tatranská Lomnica, The Slovak Republic. \\ e-mail:hric@ta3.sk, urban@ta3.sk, chochol@ta3.sk \\ 3. N. Copernicus Observatory and Planetarium, \\ 61600 Brno, The Czech Republic. e-mail: hanzld@aci.cvut.cz
}

Nova Cas 1993 (=V705 Cas) was discovered ten days before it reached its maximum brightness in the $V$ colour $\left(V_{\max }=5.53 \mathrm{mag}\right.$ and $B_{\max }=6.14$ mag) on 1993 December 17.29 (Munari et al. 1994b). The pre-nova varied around $18 \ldots 18.5 \mathrm{mag}$ and $17 \ldots 17.5 \mathrm{mag}$ in $B$ and $V$, respectively. No more prominent light fluctuations were found (Munari et al. 1994a). The nova started to fade rapidly, entering the deep minimum typical for the transition period of the DQ Her-subclass of novae in 1994 mid-February. The time development of the UV luminosity of the nova was discussed by Hauschildt et al. (1994). In this paper, we analyze our original $U B V$ photometric data and all the available data obtained by the other authors. We derive the basic parameters of the nova and present the results of our search for short-term periodicities.

Our observational material was obtained at Hlohovec, Skalnaté Pleso, Stará Lesná and Brno N. Copernicus Observatories in the interval between 1993 December 17, and 1994 December 1.

The courses of our $B$ and $V$ light curves, as well as that of the $U$ light curve, clearly confirm the membership of V705 Cas in the rather special and not so frequent subclass of novae designated as DQ Her-type. After the brightness maximum, the nova entered the early decline stage characterized by moderately fast light decrease with short-term variations (days to tens of days) superimposed on it. Approximately fifty days after the maximum, a sudden brightness decrease in all three colours is observed. The break in the light curve is well illustrated by rapid change in the course of the $U-B$ index. The nova reached the bottom of this deep minimum (16.1 mag in 
the visual) $103 \mathrm{~d}$ after the maximum. The brightness of the nova then rose again up to about $12.2 \mathrm{mag}$. The nova further oscillated irregularly by a few tenths of magnitude around this brightness up to the present.

We have used our light curves for the derivation of the following basic parameters of the nova: $t_{2, \mathrm{~B}}=40 \mathrm{~d}, t_{2, \mathrm{~V}}=33 \mathrm{~d}, t_{3, \mathrm{~B}}=63 \mathrm{~d}, t_{3, \mathrm{~V}}=61 \mathrm{~d}$. Using the formulae derived by Cohen (1985), Capaccioli et al. (1989), Schmidt (1957) and van den Bergh \& Younger (1987), we determined the following value for the mean absolute $V$ magnitude of the nova at the maximum: $M_{\mathrm{V}}=-7.05 \pm 0.22 \mathrm{mag}$. On the basis of formulae by Livio (1992) and Pfau (1976), we obtained the following mean value for the mean absolute $B$ magnitude at maximum: $M_{\mathrm{B}}=-7.03 \pm 0.20 \mathrm{mag}$. Using the empirical formula developed by Livio (1992), we derived the mass of the white dwarf component of the nova: $M_{\mathrm{WD}}=0.75 \pm 0.04 \mathrm{M}_{\odot}$. Having derived the above quantity, we were able to compute the relevant Eddington luminosity, which is $M_{\text {bol }}^{\text {Edd }}=-6.44 \pm 0.06 \mathrm{mag}$. Adopting the bolometric correction B.C. $=-0.25$, the corresponding Eddington luminosity for $V$ is $M_{\mathrm{V}}^{\mathrm{Edd}}=-6.19 \mathrm{mag}$. This means that the outburst of V705 Cas was a super-Eddington one. We obtained the mean colour excess value: $E_{\mathrm{B}-\mathrm{V}}=0.45 \pm 0.09$. The corresponding value of extinction is $1.40 \pm 0.28$, which further leads to the distance determination of $r=1.73 \pm 0.2 \mathrm{kpc}$.

We have probed the light curves of the nova during the early decline for the presence of periodicities in the period interval $0.01 \ldots 100 \mathrm{~d}$. The application of the minimization of the phase dispersion method (Stellingwerf 1978), as well as of Fourier analysis, resulted in the detection of the statistically significant period of $0.14 \mathrm{~d}$. The period could reflect either the orbital motion of the underlying binary or the rotation of the white dwarf, or else oscillations within the expanding nova envelope. Its value fits well into the observed orbital period distribution of novae.

\section{References}

Capaccioli, M., Della Valle, M., D’Onofrio, M., Rosino, L., 1989, AJ, 97, 1622

Cohen, J.G., 1985, Ap. J., 292, 90

Hauschildt, P., Starrfield, S., Shore, S., et al., 1994, AJ, 108, 1108

Livio, M., 1992, Ap. J., 393, 516

Munari, V., Hric, L., Tomov, T., Hazucha, P., 1994a, IBVS 3977

Munari, U., Tomov, T., Antonov, A., et al., 1994b, IBVS 4005

Pfau, W., 1976, A\&A, 50, 113

Schmidt, Th., 1957, Z. Astrophys., 41, 182

Stellingwerf, R.F., 1978, Ap. J., 224, 953

van den Bergh, S., Younger, P.F., 1987, A\&A, 70, 125 